\title{
Pressures Delivered By Nasal High Flow Oxygen During All Phases of the Respiratory Cycle
}

\author{
Rachael L Parke RN MHSc and Shay P McGuinness MB ChB
}

\begin{abstract}
BACKGROUND: Nasal high flow (NHF) oxygen therapy and CPAP are modes of noninvasive respiratory support used to improve respiratory function in multiple patient groups. Both therapies provide positive pressure, although this varies during the respiratory cycle. The purpose of this study was to measure and compare the airway pressure generated during different phases of the respiratory cycle in patients receiving NHF at various gas flows. METHODS: Patients scheduled for elective cardiac surgery were invited to participate. Nasopharyngeal pressure measurements were performed using $\mathrm{NHF}$ with gas flows of 30,40 , and $50 \mathrm{~L} / \mathrm{min}$. All measurements were performed in random order, with the subject breathing with mouth closed. RESULTS: During NHF the mean \pm SD nasopharyngeal airway pressures were $1.5 \pm 0.6,2.2 \pm 0.8$, and $3.1 \pm 1.2$ at 30, 40, and $50 \mathrm{~L} / \mathrm{min}$ using NHF. Analyses also determined the mean peak expiratory and mean expiratory plateau pressures. CONCLUSIONS: The expiratory pressure during NHF was higher than the mean pressure previously reported for NHF. This may account in part for the disproportional clinical effects seen with NHF. (Australian Clinical Trials Registry www.anzctr.org.au ACTRN12609000305224). Key words: airway pressure; nasal high flow oxygen; noninvasive ventilation; oxygen. [Respir Care 2013;58(10):1621-1624. (c) 2013 Daedalus Enterprises]
\end{abstract}

\section{Introduction}

Respiratory complications, including hypoxia, sputum retention, and basal lung atelectasis, remain a leading cause of post-cardiac-surgery morbidity, and can prolong hospital stay and increase costs. ${ }^{1}$ Traditionally, therapy has consisted of supplemental oxygen and noninvasive ventilation via oronasal mask to maintain adequate oxygenation in patients with inadequate postoperative respiratory function. A recent novel therapy that is gaining widespread use in the ICU and postoperative ward is nasal high flow (NHF) oxygen therapy, in which heated and humidified blended oxygen and air is administered at flows up to $60 \mathrm{~L} / \mathrm{min}$, via

The authors are affiliated with the Cardiothoracic and Vascular Intensive Care Unit, Auckland City Hospital, Auckland, New Zealand.

This study was partly supported by grant HRC11/144 and PhD scholarship 10/60/4079 from the Health Research Council of New Zealand Clinical Research Training Fellowship, to Ms Parke, and by project grant 11/25/4083 from the Green Lane Research and Education Fund. Research in the Cardiothoracic and Vascular ICU of Auckland City Hospital is partly supported by an unrestricted grant from Fisher \& Paykel Healthcare, which partly pays salaries of research nurses in the Cardiothoracic and Vascular ICU. Fisher \& Paykel Healthcare also supplied the con- a specially designed nasal interface. ${ }^{2-6}$ NHF has been demonstrated to be easy to institute and comfortable for the patient, with excellent adherence to the therapy. ${ }^{6,7} \mathrm{~A}$ recent randomized controlled trial comparing NHF to high flow oronasal mask oxygen therapy found that more patients allocated to NHF were considered to be successful on their treatment $(P=.006)$, and there was less use of noninvasive ventilation in the NHF group $(P=.10) .{ }^{2} \mathrm{How}-$ ever, until recently there was little evidence explaining the likely mechanisms of action attributed to NHF, namely, the provision of positive airway pressure, active humidification, and nasopharyngeal wash-out. ${ }^{8}$ It has previously

sumables used in this study. Ms Parke has disclosed a relationship with Fisher \& Paykel Healthcare.

Ms Parke presented a version of this paper at the International Conference of the American Thoracic Society, held May 18-23, 2012, in San Francisco, California.

Correspondence: Rachael L Parke RN MHSc, Cardiothoracic and Vascular Intensive Care Unit, Auckland City Hospital, Private Bag 92024, Auckland 1010, New Zealand. E-mail: rparke@adhb.govt.nz.

DOI: $10.4187 /$ respcare. 02358 
been demonstrated that NHF provides a low level, flow dependent positive airway pressure, $, 5,9,10$ but the clinical effect often appears to be disproportional to the low pressure provided. A common feature of all these studies is that the airway pressure reported at different NHF flows has been the mean pressure recorded over the whole of the respiratory cycle; however, observation of the pressure waveform demonstrates substantial variation in pressure during inspiration and expiration. It is plausible that the predominant benefits of positive pressure occur during expiration, particularly in patients who are at risk of, or have established, atelectasis. It could be assumed that the mean expiratory pressure may be responsible for preventing atelectasis and that the peak and mean expiratory pressures may be responsible for the re-expansion of collapsed areas.

This study aimed to quantify the pressures produced during the different parts of the respiratory cycle with NHF, using various gas flows.

\section{Methods}

This study was approved by the Northern X Regional Ethics Committee and registered with the Australian Clinical Trials Registry (www.anzctr.org.au ACTRN 12609000305224). Adult patients scheduled for elective cardiac surgery were invited to participate, and written informed consent was obtained preoperatively. Patients were excluded if there were contraindications to either NHF or noninvasive ventilation. Following surgery, and while still sedated and ventilated in the ICU, a 10 French catheter was inserted into the nasopharynx via the nose. The catheter was secured in place and remained in situ overnight. Pressure measurements were performed once the subject was awake, extubated, and sitting up in a chair, the day after surgery. Placement of the manometry catheter was first confirmed with a visual check to ensure the tip was positioned just below the uvula, and then also checked using end-tidal $\mathrm{CO}_{2}$ monitoring. If necessary, the catheter was adjusted or suctioned to obtain a clear trace. The catheter was then connected to the pressure transducer (PPT-0001 DWWW2VA-B, Honeywell, Morristown, New Jersey). This methodology was previously reported.9,10 The high flow nasal cannula system (RT033/ 034 Optiflow nasal cannula, MR880 heated humidifier, and RT241 heated delivery tube, Fisher \& Paykel Healthcare, Auckland, New Zealand) delivers humidified nasal oxygen (Fig. 1).

Measurements were performed at flows of 30,40, and $50 \mathrm{~L} / \mathrm{min}$. Measurements were performed in random order, with the subject breathing with mouth closed. The order of measurement was determined by a Latin square, constructed in a Williams design, so that each treatment occurred once per subject. ${ }^{11}$ This ensured random treat-

\section{QUICK LOOK}

\section{Current knowledge}

High-flow nasal cannula oxygen, with the gas heated and humidified to alveolar conditions, is used to treat hypoxemia and reduce ventilatory requirements. The high flow helps wash out the upper airway dead space and may provide a low level of PEEP.

\section{What this paper contributes to our knowledge}

At flows of 30-50 L/min the high-flow nasal cannula system provided a PEEP of $3-5 \mathrm{~cm} \mathrm{H}_{2} \mathrm{O}$, which may explain the observed clinical improvements. These findings also suggest the need for considering the safety of such systems with children or any condition where the cannula fits tightly in the nares.

ment allocation to each measurement, and sequences were randomly allocated to subjects square by square. A washout period was allowed between each treatment to ensure no carry-over effect. After the measurements the nasopharyngeal catheter was removed and the subject continued on the original oxygen therapy. Nasopharyngeal pressure at each flow was recorded over $1 \mathrm{~min}$ of breathing. The pressure measurements were recorded in a spreadsheet (Excel, Microsoft, Redmond, Washington) at $120 \mathrm{~Hz}$, and pressure waveforms were constructed from the data.

The mean nasopharyngeal airway pressure was determined by averaging the pressure over $1 \mathrm{~min}$, from the peak of inspiration of the first breath to the peak of inspiration of the last breath. This allowed the entire pressure profile of each breath within that minute to be included within the mean airway pressure calculation. Analysis of the inflection points of the pressure waveforms allowed determination of the start of inspiration and expiration, enabling the

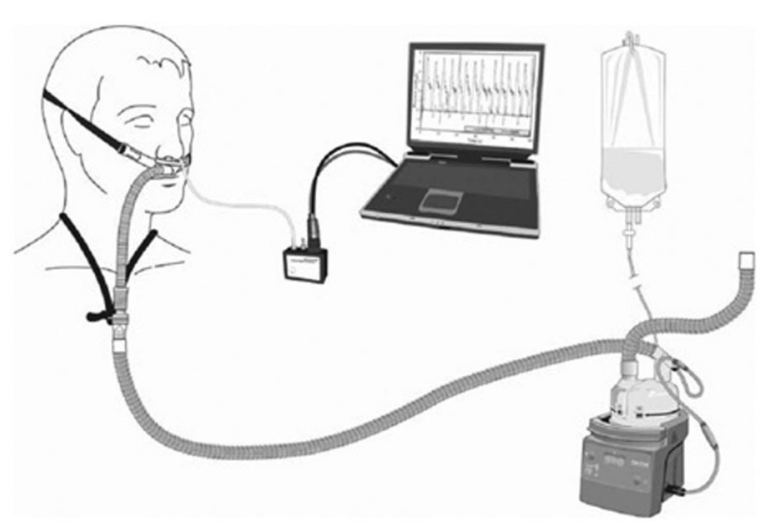

Fig. 1. Manometry setup with Optiflow nasal high flow oxygen system. 
Table 1. Subject Characteristics $(n=15)$

\begin{tabular}{lc}
\hline \hline Age, mean \pm SD y & $59.5 \pm 10.6$ \\
Male, no. & 14 \\
Female, no. & 1 \\
Height, mean \pm SD cm & $173 \pm 4$ \\
Weight, mean \pm SD kg & $85.1 \pm 17$ \\
Body mass index, mean \pm SD kg/m ${ }^{2}$ & $29 \pm 5$ \\
Ethnicity, no. & \\
$\quad$ New Zealand European & 10 \\
New Zealand Maori & 3 \\
Pacific Islander & 2 \\
\hline
\end{tabular}

calculation of peak expiratory pressure, average expiratory pressure, average inspiratory pressure, and average plateau pressure. All data analysis was performed with spreadsheet software (Excel, Microsoft, Redmond, Washington). Data are presented as mean $\pm \mathrm{SD}$.

\section{Results}

Fifteen patients scheduled for elective cardiac surgery participated (Table 1). Thirteen subjects had coronary artery bypass surgery. Two subjects had valve surgery. All the surgeries were via median sternotomy and involved cardiopulmonary bypass.

Table 2 presents the mean \pm SD nasopharyngeal airway, expiratory plateau, peak expiratory, mean expiratory and mean inspiratory pressures during NHF. The mean \pm SD nasopharyngeal airway pressures were $1.52 \pm 0.6$, $2.21 \pm 0.8$, and $3.1 \pm 1.2 \mathrm{~cm} \mathrm{H}_{2} \mathrm{O}$ at 30,40 , and $50 \mathrm{~L} / \mathrm{min}$. Analysis of the pressure generated during different parts of the respiratory cycle demonstrated that higher pressures are obtained during expiration than during the other parts of the respiratory cycle, and that both the expiratory plateau pressure and the peak expiratory pressure are flow dependent (see Table 2).

All the individual measurements and the mean nasopharyngeal pressures are shown in Figure 2. Typical pressure profiles from one subject are shown in Figure 3.

\section{Discussion}

This study describes the airway pressures generated at 3 flows in subjects receiving NHF and reports for the first time the pressure delivered during different phases of the respiratory cycle by NHF. Previous work ${ }^{9,10}$ reported the mean pressure delivered by NHF across the whole of the respiratory cycle, but because these pressures were low (mean 2-4 $\mathrm{cm} \mathrm{H}_{2} \mathrm{O}$ ), doubt has been raised about the clinical relevance of positive pressure as a mechanism of effect of NHF.

The airway pressure waveforms demonstrate the pressure variability during the different phases of the respiratory cycle during NHF; the pressure is higher during the expiratory phase. It has been hypothesized that the PEEP created by NHF reduces the work of breathing and improves oxygenation. . $^{5,12}$

The present study shows that the peak and expiratory plateau pressures during NHF are higher than the mean airway pressure previously reported, and we suggest that this may help explain the clinical benefits of NHF. NHF at flows up to $50 \mathrm{~L} / \mathrm{min}$ provides PEEP and peak expiratory pressure at significantly higher levels than the recorded mean pressure, but these are still less than that recorded and delivered with mask CPAP. We suggest that in patients who require an escalation in respiratory support, NHF should be seen as a logical step between traditional oxygen therapy and mask CPAP. Similarly, NHF is a logical intermediary step when weaning patients from higher positive airway pressure systems to low flow oxygen therapy. ${ }^{13}$

It has also been proposed that the high flow with NHF creates resistance to exhalation and clinically important positive airway pressure when the patient breathes with mouth closed. ${ }^{14}$ This pressure effect may then be transmitted down the airways to the alveoli, assisting in reexpansion of atelectatic areas. This increased resistance during expiration creates an expiratory positive airway pressure not dissimilar to that employed in devices for the management of obstructive sleep apnea. ${ }^{15}$ This increased positive airway pressure may be responsible for the im-

Table 2. Airway Pressures Delivered With Nasal High Flow Oxygen

\begin{tabular}{|c|c|c|c|c|c|}
\hline $\begin{array}{l}\text { Flow } \\
\mathrm{L} / \mathrm{min}\end{array}$ & $\begin{array}{l}\text { Airway Pressure } \\
\quad\left(\mathrm{cm} \mathrm{H}_{2} \mathrm{O}\right)\end{array}$ & $\begin{array}{c}\text { Average Plateau } \\
\text { Pressure } \\
\left(\mathrm{cm} \mathrm{H}_{2} \mathrm{O}\right)\end{array}$ & $\begin{array}{c}\text { Peak Expiratory } \\
\text { Pressure } \\
\left(\mathrm{cm} \mathrm{H}_{2} \mathrm{O}\right)\end{array}$ & $\begin{array}{c}\text { Average Expiratory } \\
\text { Pressure } \\
\left(\mathrm{cm} \mathrm{H} \mathrm{H}_{2} \mathrm{O}\right)\end{array}$ & $\begin{array}{c}\text { Average Inspiratory } \\
\text { Pressure } \\
\left(\mathrm{cm} \mathrm{H}_{2} \mathrm{O}\right)\end{array}$ \\
\hline 30 & $1.52 \pm 0.6$ & $1.71 \pm 0.73$ & $3.01 \pm 1.18$ & $2.1 \pm 0.83$ & $0.55 \pm 0.38$ \\
\hline 40 & $2.21 \pm 0.8$ & $2.48 \pm 0.94$ & $3.81 \pm 1.45$ & $2.88 \pm 1.04$ & $1.11 \pm 0.51$ \\
\hline 50 & $3.10 \pm 1.2$ & $3.41 \pm 1.24$ & $4.86 \pm 1.79$ & $3.81 \pm 1.33$ & $1.77 \pm 0.69$ \\
\hline
\end{tabular}

$\overline{\text { Values are mean } \pm \mathrm{SD}}$ 


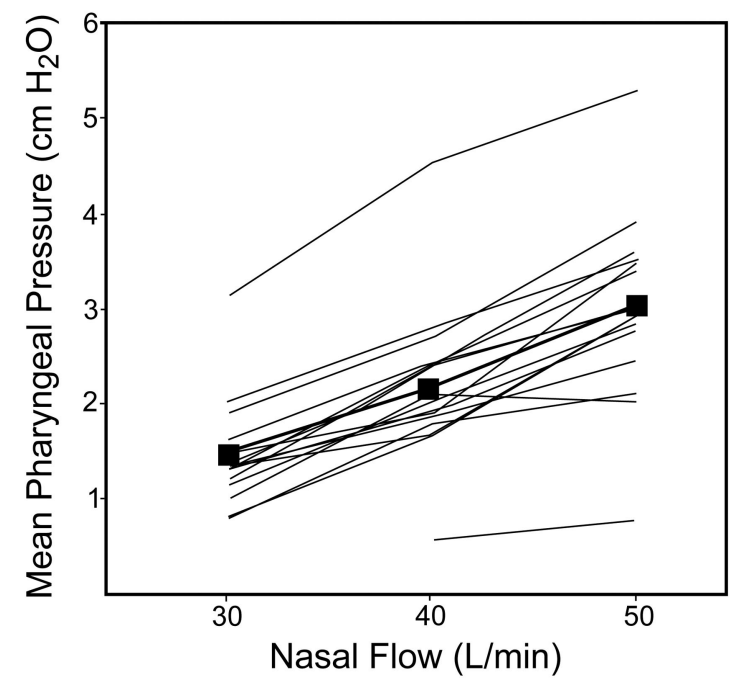

Fig. 2. Individual and mean nasopharyngeal airway pressures delivered with Optiflow nasal high flow oxygen system.

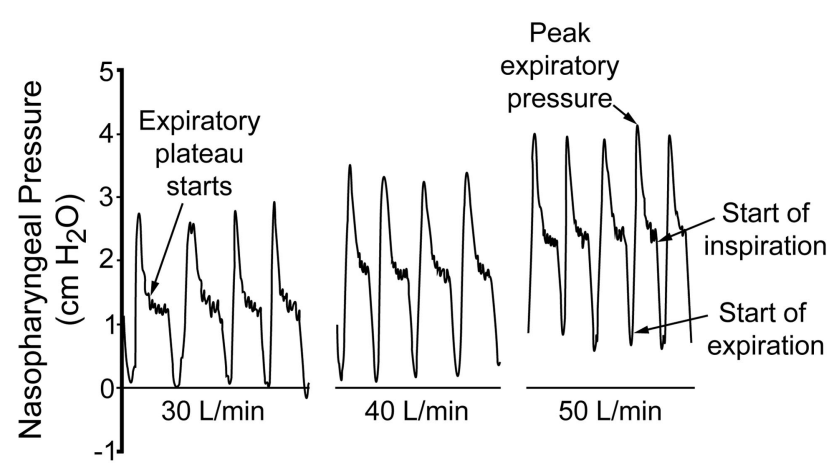

Fig. 3. Pressure profiles from one subject at increasing gas flows with the Optiflow nasal high flow oxygen system.

proved results seen when NHF is employed for hypoxemic respiratory distress. ${ }^{2,12}$

\section{Limitations}

This study was designed to measure respiratory pressures, not physiological outcomes, so no data were collected on breathing frequency, $\mathrm{S}_{\mathrm{pO}_{2}}$, minute ventilation, or lung volumes. Only one of our subjects was female, so our results may not be entirely applicable to female patients, and we were unable to test for sex differences. However, female patients experience significantly higher airway pressure than males with Optiflow. ${ }^{16}$ Also, due to the nature of the patient population available, our cohort included only adult patients following cardiac surgery, so our results may not be generalizable to all patients, such as pediatric patients.

\section{Conclusions}

The pressure generated by NHF during expiration was higher than the mean airway pressure over the whole respiratory cycle. Although NHF is unable to provide pressure similar to CPAP, this study shows that the mean and peak expiratory pressures are in a range that is likely to have a clinical effect, so we believe that positive airway pressure is one of the mechanisms of NHF's clinical benefits. Further work is required to elucidate NHF's mechanisms of action, including its effects on work of breathing, lung volumes, oxygenation, intrathoracic pressure, and lung recruitment.

\section{REFERENCES}

1. Weissman C. Pulmonary complications after cardiac surgery. Semin Cardiothorac Vasc Anaesth 2004;8(3):185-211.

2. Parke R, McGuinness SP, Eccleston ML. A preliminary randomized controlled trial to assess effectiveness of nasal high-flow oxygen in intensive care patients. Respir Care 2011;56(3):265-270.

3. Zarbock A, Mueller E, Netzer S, Gabriel A, Feindt P, KindgenMilles D. Prophylactic nasal continuous positive airway pressure following cardiac surgery protects from postoperative pulmonary complications. Chest 2009;135(5):1252-1259.

4. Parke R, McGuinness S, Dixon R, Jull A. Protocol for a randomised controlled trial of nasal high flow oxygen therapy compared to standard care in patients following cardiac surgery: the HOT-AS study. Int J Nurs Stud 2012;49(3):338-344.

5. Corley A, Caruana L, Barnett A, Tronstad O, Fraser JF. Oxygen delivery through high-flow nasal cannulae increase end-expiratory lung volume and reduce respiratory rate in post-cardiac surgical patients. Br J Anaesth 2011;107(6):998-1004.

6. Nicolet J, Poulard F, Baneton D, Rigal JC, Blanloeil Y. [High-flow nasal oxygen for severe hypoxemia after cardiac surgery]. Ann Fr Anesth Réanim 2011;30(4):331-334. Article in French.

7. Tiruvoipati R, Lewis D, Haji K, Botha J. High-flow nasal oxygen vs high-flow face mask: a randomized crossover trial in extubated patients. J Crit Care 2010;25(3):463-468.

8. Dysart K, Miller TL, Wolfson MR, Shaffer TH. Research in high flow therapy: mechanisms of action. Respir Med 2009;103(10):1400-1405.

9. Parke R, McGuinness S, Eccleston M. Nasal high-flow therapy delivers low level positive airway pressure. Br J Anaesth 2009;103(6): 886-890.

10. Parke R, Eccleston ML, McGuinness SP. The effects of flow on airway pressure during nasal high-flow oxygen therapy. Respir Care 2011;56(8):1151-1155.

11. Jones B, Kenward M. Design and analysis of cross-over trials. London: Chapman and Hall; 1989.

12. Roca O, Riera J, Torres F, Masclans J. High-flow oxygen therapy in acute respiratory failure. Respir Care 2010;55(4):408-413.

13. Masclans JR, Roca O. High-flow oxygen therapy in acute respiratory failure. Clin Pulm Med 2012;19(3):127-130.

14. Ritchie JE, Williams AB, Gerard C, Hockey H. Evaluation of a humidified nasal high-flow oxygen system, using oxygraphy, capnography and measurement of upper airway pressures. Anaesth Int Care 2011;39(6):1103-1110.

15. McGinley B, Patil S, Kirkness J, Smith P, Schwartz A, Schneider H. A nasal cannula can be used to treat obstructive sleep apnea. Am J Respir Crit Care Med 2007;176(2):194-200.

16. Groves N, Tobin A. High flow nasal oxygen generates positive airway pressure in adult volunteers. Aust Crit Care 2007;20(4):126131. 